\title{
Investigation of Anti-Infection Mechanism of Lactoferricin and Splunc-1
}

\author{
Yung An Tsou, ${ }^{1,2}$ Hung-Jin Huang, ${ }^{3}$ Wesley Wen Yang Lin, ${ }^{4}$ and Calvin Yu-Chian Chen ${ }^{2,5}$ \\ ${ }^{1}$ Otolaryngology Head and Neck Surgery, China Medical University Hospital, Taichung 40402, Taiwan \\ ${ }^{2}$ School of Medicine, College of Medicine, China Medical University, Taichung 40402, Taiwan \\ ${ }^{3}$ Department of Chinese Pharmaceutical Sciences and Chinese Medicine Resources, College of Pharmacy, China Medical University, \\ Taichung 40402, Taiwan \\ ${ }^{4}$ Department of Biological Science and Technology, National Chiao Tung University, Hsinchu 30010, Taiwan \\ ${ }^{5}$ Department of Biomedical Informatics, Asia University, Taichung 41354, Taiwan
}

Correspondence should be addressed to Calvin Yu-Chian Chen; ycc929@MIT.edu

Received 15 January 2014; Revised 8 February 2014; Accepted 8 February 2014; Published 30 April 2014

Academic Editor: Fuu-Jen Tsai

Copyright (c) 2014 Yung An Tsou et al. This is an open access article distributed under the Creative Commons Attribution License, which permits unrestricted use, distribution, and reproduction in any medium, provided the original work is properly cited.

\begin{abstract}
The innate immune system is the first line in the defense system and prevents the body from further bacteria, virus, or fungal infections. Most of the innate immune system is relevant to mucosa immunity. Lactotransferrin is secreted from the human mammal breast duct epithelial tissue and strengthens infant immunity to defense with regard to outward pathogens. Splunc-1 is also an innate material secreted from the soft palate, lung, nasal cavity epithelium, and mucosa. It helps with mucosa defense against bacterial, virus, and even fungus. LPS is the main etiology of Gram-negative bacilla infection source. And studies of lactoferricin and slpunc1 both can combine with LPS and subsequently cause insults to the mucosa. Although, we know that both of them partake in an important role in innate immunity, we do not know the effects when they work together. In this study, we just overview silicon stimulation to examine the combination of Lactoferricin and Splunc-1 and the effect with regard to LPS.
\end{abstract}

\section{Introduction}

Splunc-1 is secreted by the soft palate, lung, and nasal cavity epithelium and is considered an innate material to help mucosa immunity [1]. There have been a number of studies which have proven the antibacterial benefits [2] with regard to Staphylococcus aureus, Streptococcus, Pseudomonas $[3,4]$, mycoplasma pneumonia [5], and klebsiella pneumonia [6]. It is also considered to have antibiofilm functionality through a reduction of the surface tension by regulating the airway surface liquid volume $[7,8]$. It is also very important connector to coordinate innate and adaptive immunity [9]. Furthermore, it is quite important to protect the upper airway from infections [10] and to neutralize pathogens containing lipopolysaccharides (LPS).

LPS is the major component of Gram-negative bacteria (GNB) outer membrane and it belongs to lipoglycans, comprising a lipid and a polysaccharide connected by a covalent bond. Many kinds of GNB have this crucial pathogenic material and lead to human infections.

Lactoferricin is an iron-binding glycoprotein not only found in milk or human mammal ducts but also found in exocrine secretions such as nasal secretions, tears, saliva, urine, uterine secretions, and amniotic fluids and is considered a crucial innate immune material [11]. Bovine lactoferricin has even stronger antimicrobial function [12]. We examined the cyclic domain of short derivatives within Bovine lactoferricin, which has the power to protect against $E$. coli, S. aureus, and C. albicans [13]. It can also bind to LPS and reduce the inflammation and infection condition via inhibition of IL-1 and LPS activity in the intervertebral disc [14].

In addition, bactericidal effects were found in lactoferricin material by their binding ability to LPS in Gram-negative bacteria and stabilized cell membranes 


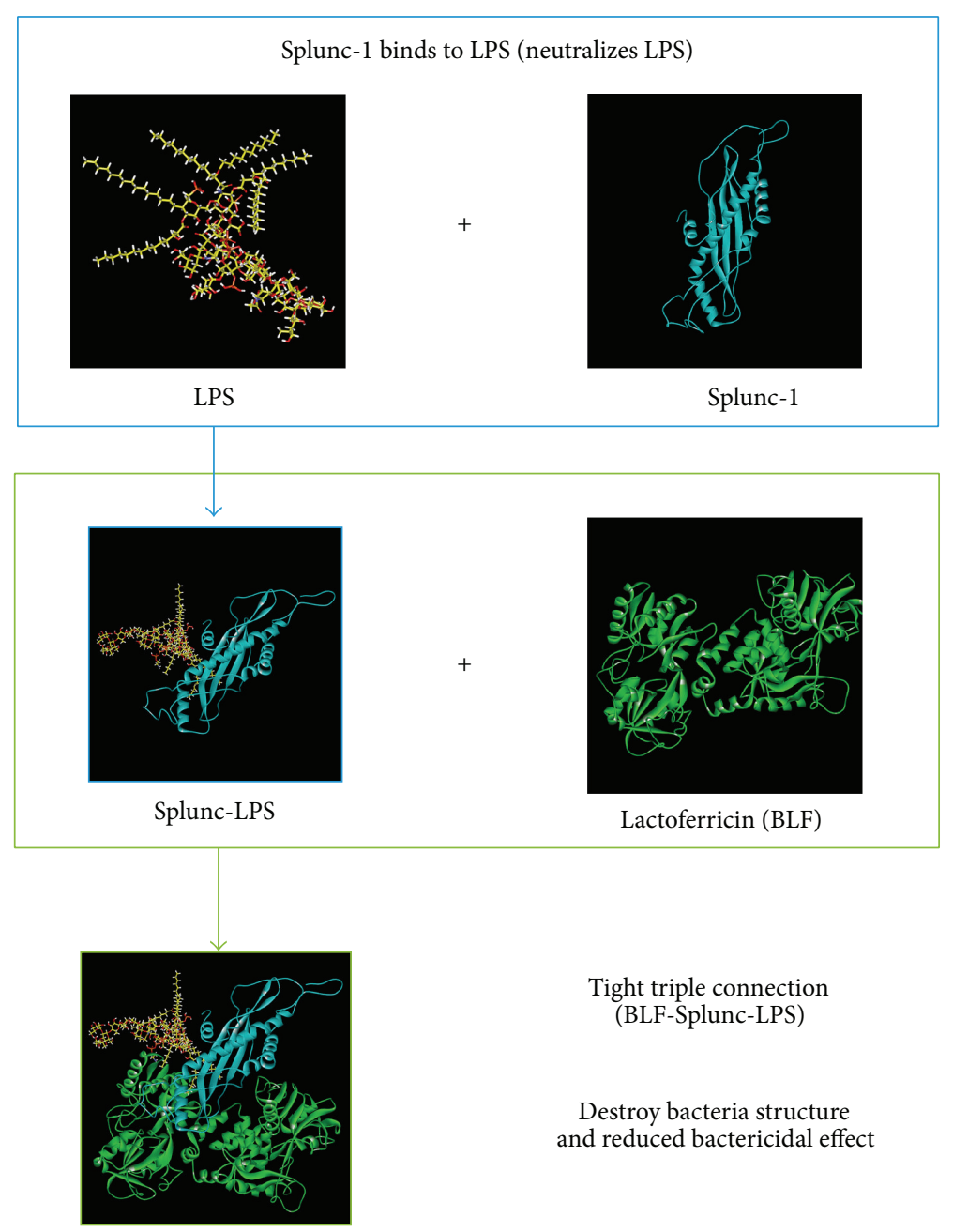

FIGURE 1: Study hypothesis.

$[15,16]$. The upper airway (nasal sinus) biofilm formation is also correlated to a reduced level of lactoferricin [17]. Chronic rhinosinusitis and infection correlate not only to decreased lactoferricin but also to decreased Splunc-1 expression [18].

Since these new silicon findings can help pharmaceutical companies to develop innovative drugs to reduce the biofilm formation in the airway to reduce further comorbidities by outward human pathogens [19], therefor, we used computeraided drugs design according to many research for further investigation [20-24]. In silico drug design includes database virtual screening [25-30], quantitative structureactivity relationship [31-34], protein-protein interactions [35], and molecular dynamics simulation [36-39], and these approaches are based on theory [40], web server [41], compound database [42], and risk targets [43-47] to perform studies of drug design or identify mechanism. Since both lactoferricin and Splunc-1 are important to protect human from LPS related infection, we hypothesize that the two proteins maybe combine to treat LPS related upper airway infections and might have synergistic effects to prevent human upper airway infection diseases as intractable sinusitis, tonsillitis, and severe pneumonia. (Figure 1). Therefore, we can potentially invent new drugs or nasal sprays to send these two important innate immune materials to the nasal cavity by nasal spray and send them to the lower airway by inhaler.

\section{Materials and Methods}

2.1. Structure Preparation. The protein structure of Splunc1 was obtained from UniProt database; we used an I-TASSER server [48-50] (iterative threading assembly refinement algorithm) to build the $3 \mathrm{D}$ structure of Splunc-1 protein from the amino acid sequence. We utilized Ramachandran plot [51] and 3D profile [52] to validate the 3D structure of Splunc-1. We choose the most reliable prediction structure for further protein-protein interaction survey. X-ray crystal structure of Bovine lactoferricin was achieved from the PDB database (PDB:1BLF) [53]. 2D structure of LPS is gained from PubChem Compound database. We utilized MM2 force 


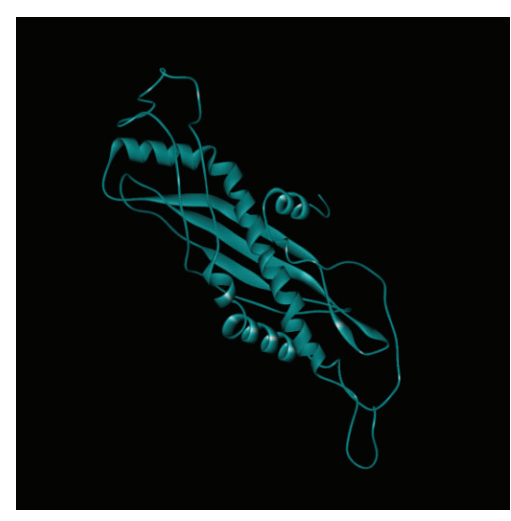

Model 1: C-score $=-1.92$

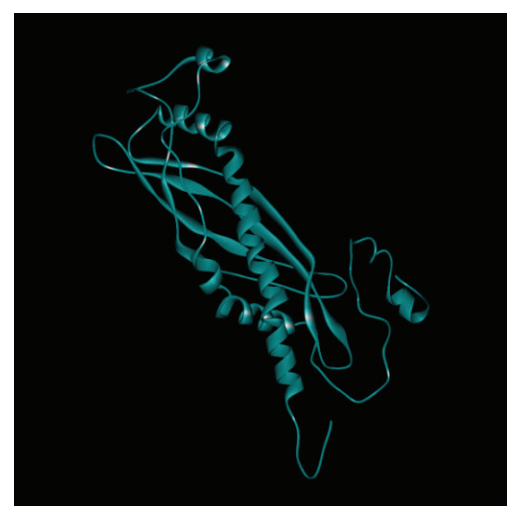

Model 3: C-score $=-2.73$

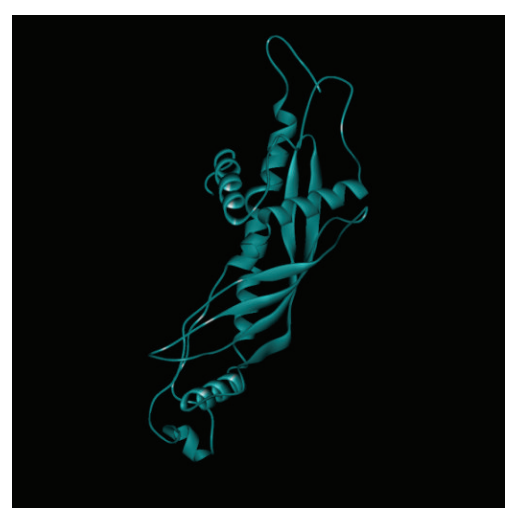

Model 2: C-score $=-2.52$

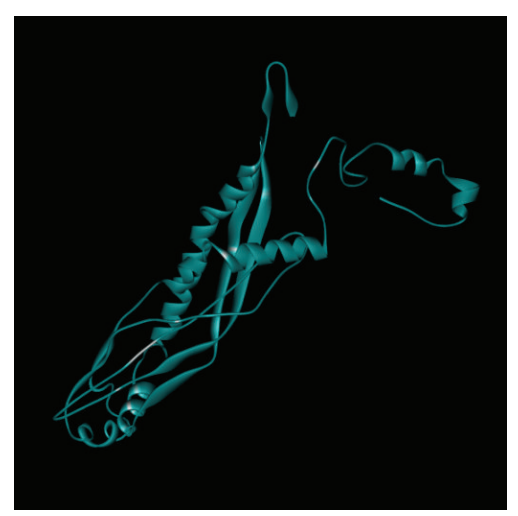

Model 4: C-score $=-3.59$

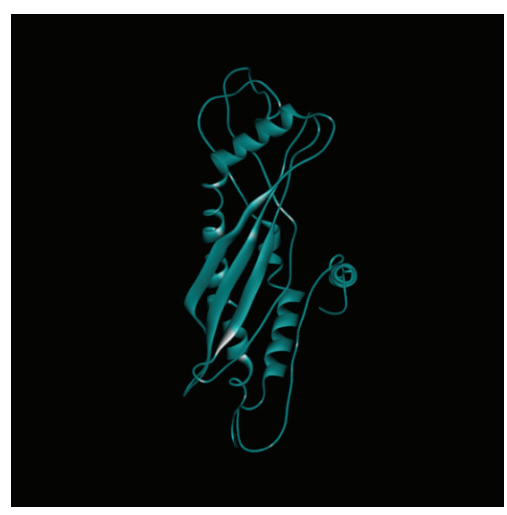

Model 5: C-score $=-3.10$

FIGURE 2: Five structures stimulated by I-TASSER; C-score shows that the reasonable ranges involved scores from -5 to 2 , and the higher score renders a more reliable structure.

field [54] of ChemBioOffice 2010 software to optimize and calculate the $3 \mathrm{D}$ compound structure.

2.2. ZDOCK and LibDock Analysis. ZDOCK [55] and LibDock [56] programs in the Accelrys Discovery Studio 2.5.5.9350 (DS 2.5) [52] were used for protein and compound interaction. ZDOCK was employed to simulate Splunc-1 and Bovine lactoferricin interaction. Angular step size was set as 6 to analyze 54,000 possible proteins interaction poses; ZDOCK score was calculated by ZDOCK program, according to the shape complementarity of the protein complexes. LibDock is used to build protein-ligand interaction. The possible connecting methods of Splunc-1-lactoferricin complex to LPS are analyzed by LibDock program. We used the sphere of $40 \AA$ radiuses as the binding area; polar and nonpolar molecules are regarded as hotspots for active site definition. The number of hotspots was set as 100 for conformer matching. The conformation method was using FAST mode. The final predicted protein-ligand complex 
TABLE 1: Top ten ranking of docking binding affinity of Splunc-1 and lactoferricin.

\begin{tabular}{lc}
\hline Pose number & ZDOCK score \\
\hline Pose 1 & 24.84 \\
Pose 2 & 24.78 \\
Pose 3 & 24.6 \\
Pose 4 & 23.8 \\
Pose 5 & 23.7 \\
Pose 6 & 23.7 \\
Pose 7 & 23.64 \\
Pose 8 & 23.6 \\
Pose 9 & 23.5 \\
Pose 10 & 23.38 \\
\hline
\end{tabular}

was optimized by energy minimization, and the mode of minimization was set as smart minimization. CHARMM [57] force field was used for calculation in energy minimization.

2.3. Molecular Dynamics Simulations. The molecular dynamics simulation for protein-ligand complexes is analyzed by Standard Dynamics Cascade of DS 2.5 [52]. Energy minimization process is performed using two stages of minimizations by steepest descent and conjugate gradient $[58,59]$, and we do heating stimulation and set the system temperature increasing from $50 \mathrm{~K}$ to $310 \mathrm{~K}$ in $50 \mathrm{ps}$ and then maintain in $310 \mathrm{~K}$ for 200 ps. Afterward, we performed constant temperature dynamics environment for $40 \mathrm{~ns}$ (NVT type) for production simulation. We set the decay time for temperature coupling as 0.4 . Trajectory analysis for conformations changes survey is done by analyzing production simulation saved data by every 10,000 ps. The distancedependent dielectrics method was utilized for solvent model. The time period for each simulation step was set as $2 \mathrm{fs}$. We constrain all bonds containing hydrogen by employing SHAKE algorithm [60] in the system during simulation.

\section{Results}

The amino acid is used for predicting possible five protein structures by I-TASSER server (Figure 2); all the C-score numbers are in the reasonable range; the higher score rendered more reliable protein structure. Then we validated the simulated Splunc-1 structural residues in their docking regions by Ramachandran plot, and $92.5 \%$ were distributed at a reliable region which was $79.5 \%$ and $13.0 \%$ of residues in favored and allowed regions, respectively, as shown in Figure 3. In Figure 4 we showed Splunc-1 residues by 3Dprofile analysis, and valid residues were proven by most of the validation scores being above zero.

Therefore, the connection of Bovine lactoferricin and LPS was based upon Model 1 structure for further research and poses survey. We further analyzed interactions between LPS and Splunc-1-Bovine lactoferricin complex by ZDOCK. We choose the top ten docking score for LPS binding for Splunc1-Bovine lactoferricin complex (Table 1). We found that the LPS could be connected to the Splunc-1-Bovine lactoferricin

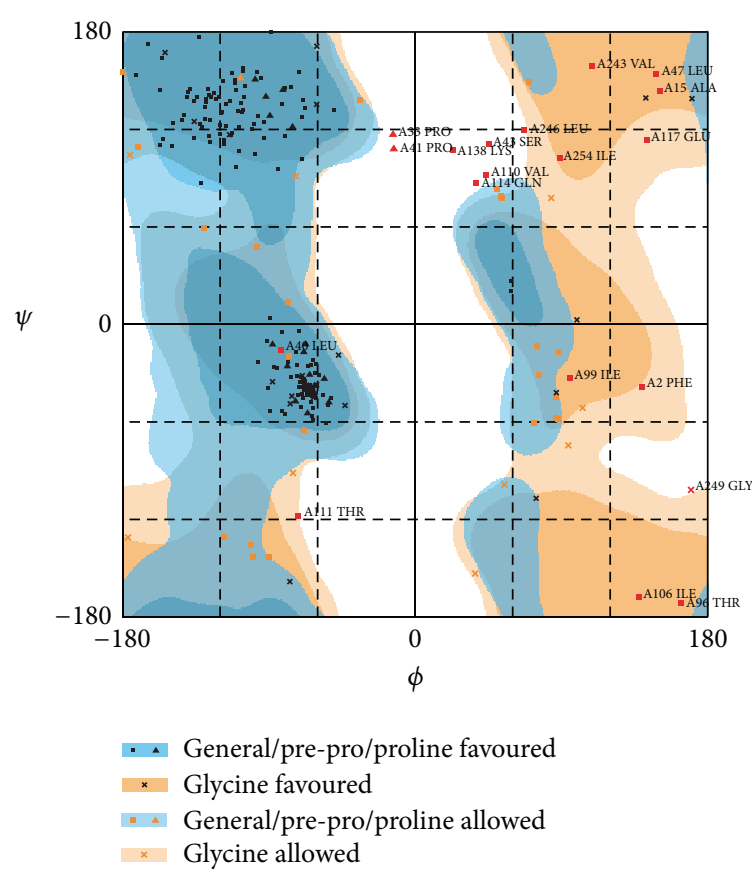

Number of residues in favoured region ( $98.0 \%$ expected): $202(79.5 \%)$ Number of residues in allowed region ( 2.0\% expected): $33(13.0 \%)$

Number of residues in outlier region: 19 (7.5\%)

FIGURE 3: Ramachandran plot of Splunc-1 residues for structure validation, $79.5 \%$ and $13.0 \%$ residues in favored and allowed regions, respectively.

complex (Pose 6) by LibDock and the LibDock score was 180.368 (Figure 5). Prior study indicated that Splunc-1-Bovine lactoferricin complex could connect to LPS, and we also proved that Splunc-1 and Bovine lactoferricin could connect to the LPS under LibDock program. And the LibDock score was 128.051 for PLUNC and LPS interaction and 64.152 for lactoferricin and LPS interaction (Figure 6). In addition, we found the Splunc-1 and Bovine lactoferricin complex indeed does have synergistic effect in connecting LPS. In further study, MD simulation (molecular dynamics simulation) in DS 2.5 was carried out to simulate the protein-protein and protein-ligand complexes. In addition, the stability of the binding poses in dynamic conditions was analyzed.

\section{Discussion}

The upper airway organs including the nasal cavity, nasal sinus, pharyngeal mucosa, palatine, and lingual tonsils are the first defense organs that prevent humans from suffering further damage from bacteria, virus, and fungus and upper airway mucosa is also considered a part of innate immunity, especially regarding pathogens comprising LPS [61]. Lactoferricin and Splunc-1 are two very crucial materials for innate immunity secreted from the mammal glandular epithelium and upper airway $[1-4,11,14,62]$.

Persistent sinusitis related to biofilm formation also revealed low levels of lactoferricin and Splunc-1 $[4,17,18]$. 


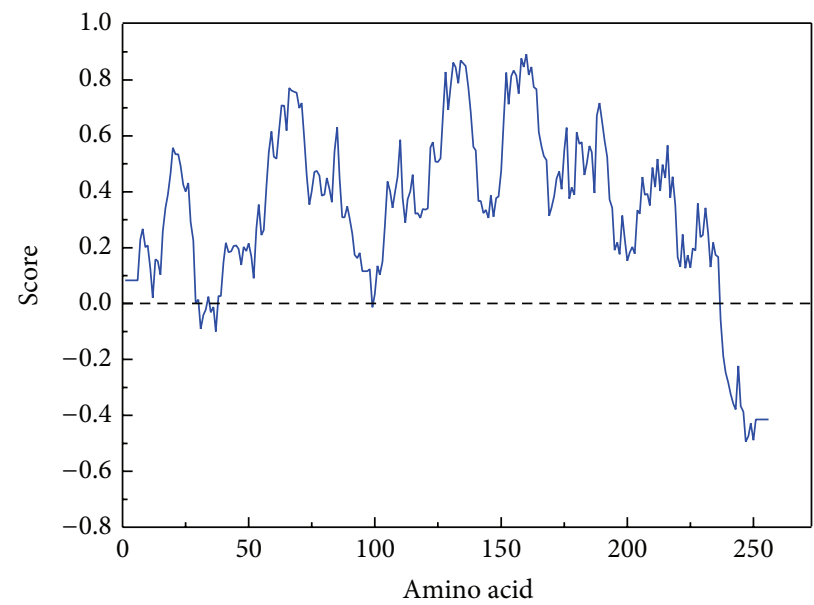

FIGURE 4: 3D-profile analysis of SPLUNC residues; the verified score above zero indicated valid residues.

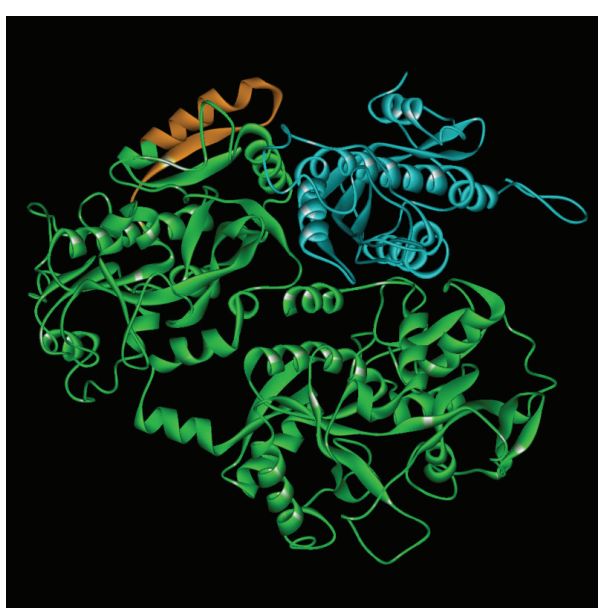

(a)

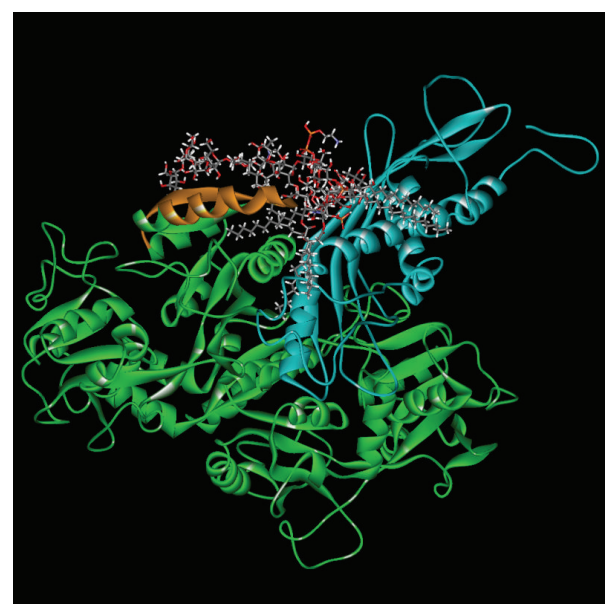

(b)

FIgURE 5: (a) The results of the connection of Splunc-1 (blue) and Bovine lactoferricin (green) (Pose 6 from ZDOCK); the orange part refers to the Bovine lactoferricin. (b) By the LibDock result, the Splunc-1 and Bovine lactoferricin complex could connect to the LPS (LibDock score $=180.368$ ).

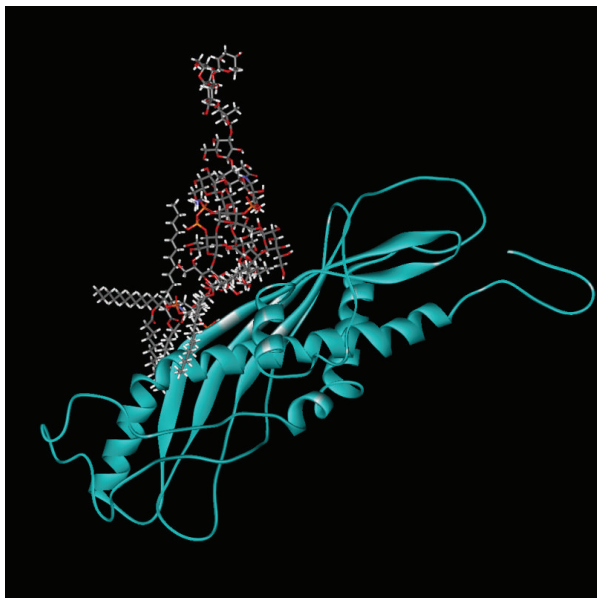

(a)

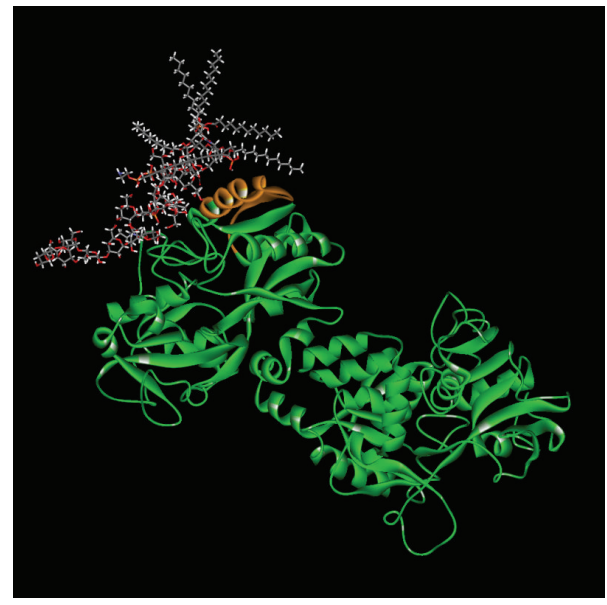

(b)

FIgURE 6: (a) Splunc-1 and (b) Bovine lactoferricin and their connected pose to LPS. The LibDock scores were 128.051 and 64.152. 


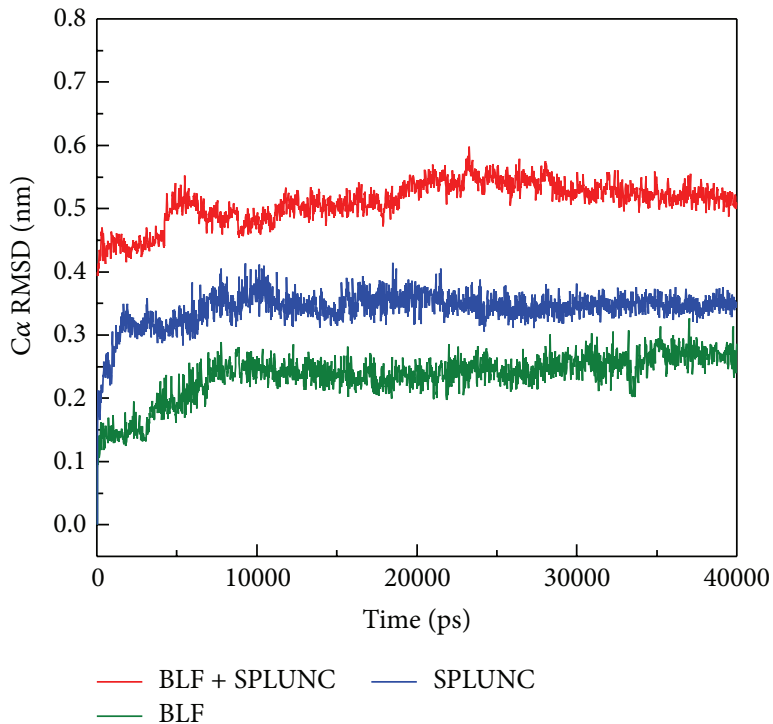

(a)

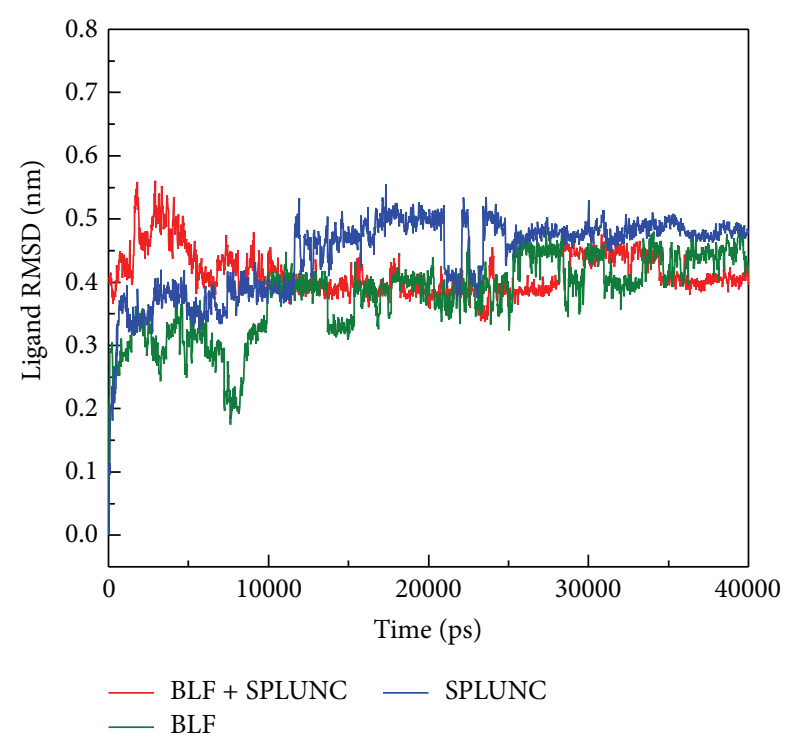

(b)

FIGURE 7: RMSD analysis for BLF, SPLUNC, and protein-protein complex (BLF and SPLUNC) among all simulation times. (a) RMSD value of the $\mathrm{C} \alpha$ of all proteins. (b) RMAD value of lipopolysaccharide in the protein binding site.

Low levels of lactoferricin in the sinus mucosa area are more susceptible to biofilm formation [17]. The pathogen Pseudomonas and Staphylococcus aureus harbored in the human nasal sinuses are considered to be correlated to sinus biofilm formation and also showed a decreased Splunc-1 expression [63]. Chronic rhinosinusitis related to biofilm formation is very difficult to treat with penicillin and some studies have shown lactoferricin could be helpful and that it has a synergic effect with regard to penicillin resistant Pseudomonas infection [64]. Therefore, combine use innate immune materials as lactoferricin and Splunc-1 may partake in an important role for eradicating biofilm formation and preventing patients from further surgical treatment for intractable sinusitis or tonsillitis.

This is also worthy to be investigated for treating the human infection diseases related to biofilm in the future.

Although there have been studies concerning lactoferricin and Splunc-1 being used to suppress LPS related infection $[2,14,61]$, there have been no studies concerning combining the two innate materials to combat LPS related infections. We found the two materials could both bind to LPS and form more stabilized compounds binding to LPS. In Figure 2, we selected the possible structure for Splunc-1 for further docking analysis and their docking power was also shown in Table 1. Then the docking of Splunc-1 to LPS and the docking of Lactoferricin, Splunc1 , and LPS are shown in Figure 5 and these three could be bound well in silicon simulation. In addition, the two innate immune materials could bind to LPS separately well as seen in Figure 6; each of them has good binding ability to LPS and this has also been proven in many human studies.
Unfortunately, there have been no prior studies concerning these two as a combined effect to neutralize LPS in the related literature. Therefore we conducted this study to assist in considering possible future drug invention to intensify innate immune deficient patients in a silicon model first and simple culture based TEM survey. Based on RMSD analysis for BLF, SPLUNC, and protein-protein complex (BLF and SPLUNC) with $40 \mathrm{~nm}$ simulation time, we confirm that the BLF-Splunc1-LPS combination is the most stable one (Figure 7). The energy analysis for BLF, SPLUNC, and protein-protein complex (BLF and SPLUNC) with LPS during $40000 \mathrm{ps}$ simulation showed the lowest total energy, Van der Waals (VDW) energy, and electrostatic energy (Figure 8). Radius of gyration analysis (Figure 9) and mean square displacement (MSD) (Figure 10) of atoms for atom migration assay both showed stable binding of protein-protein complex (BLF and SPLUNC) to LPS even after $40 \mathrm{~ns}$ of simulation. The migration of BLF-Splunc-LPS complex is less than BLF alone binding with LPS. Thus, the BLF-Splunc-LPS complex is more stable than BLF-LPS complex and suggests the Splunc help the BLF binding to LPS. However, we still need studies to survey if there were any synergic effects of the BLF and Splunc.

\section{Conclusion}

Splunc-1 is considered to be a material mainly secreted from the upper airway. Lactoferricin is also considered to have a wide antimicrobial activity against bacterial, viral, and fungal pathogens. Both of them can contribute to immune function and can be found in the upper airway. We also found both of them could have stable binding to LPS related pathogens. 


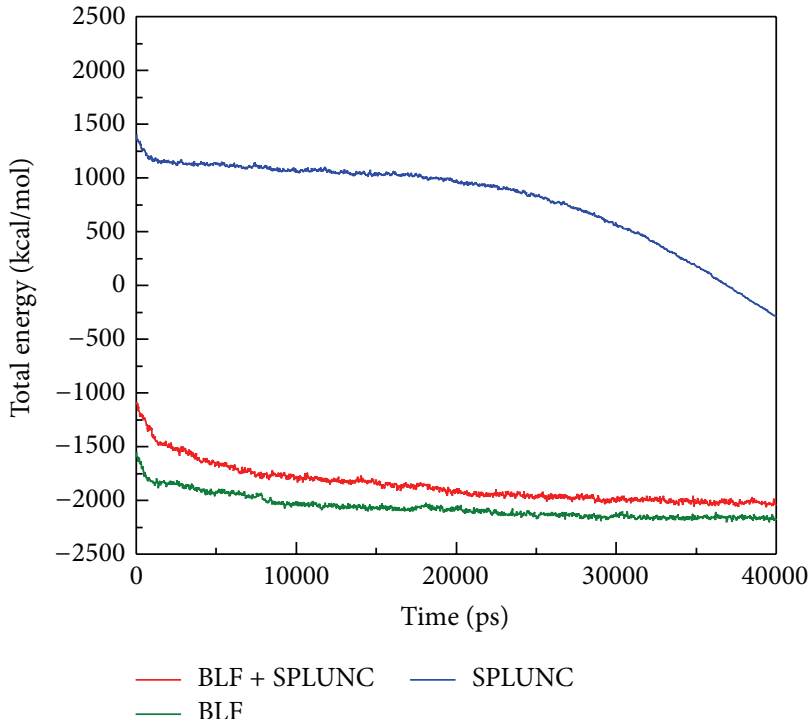

(a)

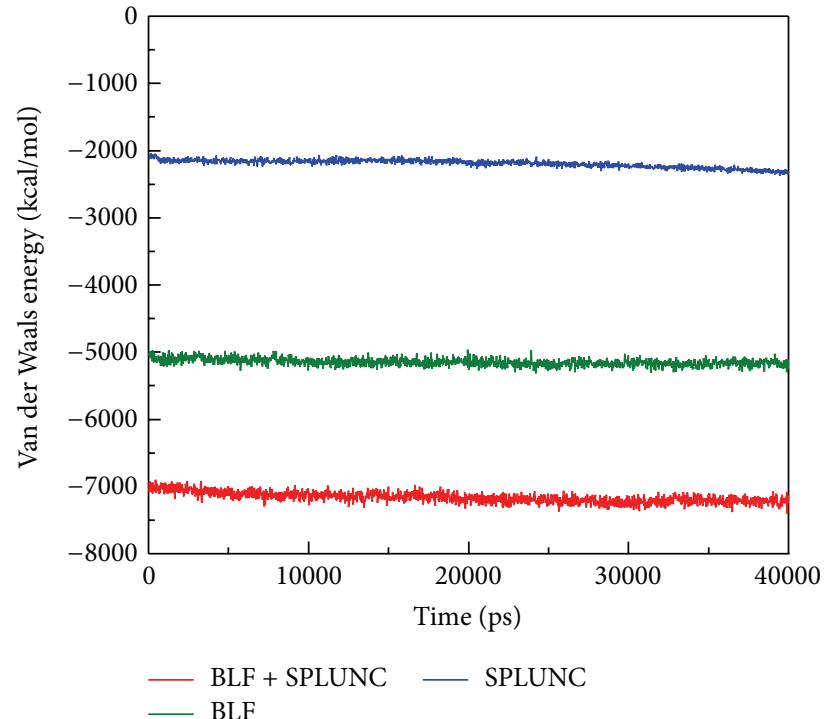

(b)

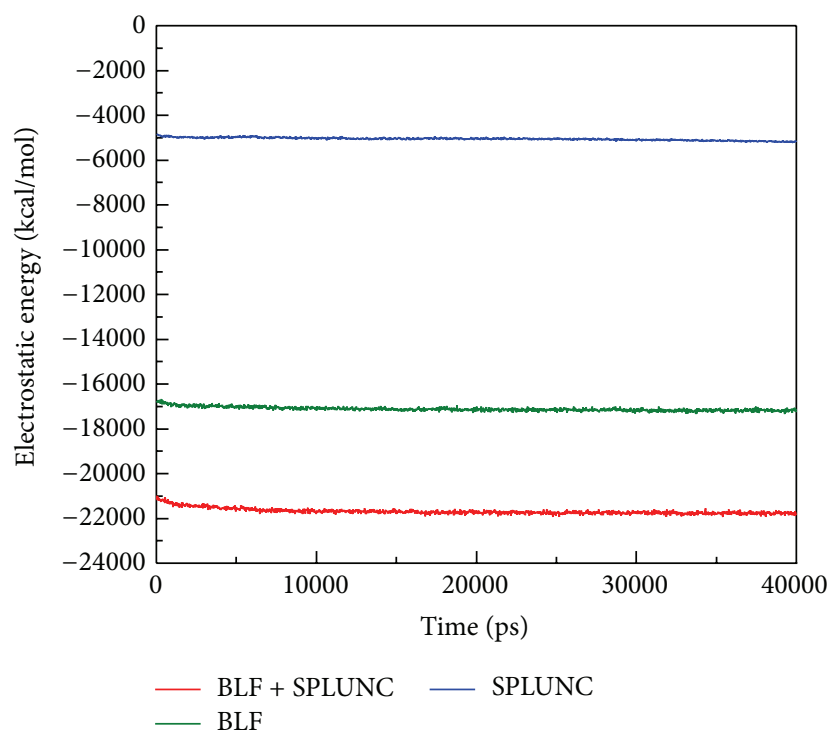

(c)

FIGURE 8: Energy analysis for BLF, SPLUNC, and protein-protein complex (BLF and SPLUNC) during 40000 ps simulation: (a) total energy, (b) Van der Waals (VDW) energy, and (c) electrostatic energy.

Based on these findings it is promising to develop new drug or find new drugs and deliver them by the nasal cavity or oral spray or even orally to strengthen innate immunity and prevent comorbidities caused by pathogen related infections in the future.

\section{Conflict of Interests}

The authors affirm that there is no conflict of interests to declare.

\section{Authors' Contribution}

Yung An Tsou and Hung-Jin Huang contributed equally to this work.

\section{Acknowledgments}

The research was supported by Grants from the National Science Council of Taiwan (NSC102-2325-B039-001, NSC1022221-E-468-027-, and NSC101-2314-B-039-013-MY3), Asia 


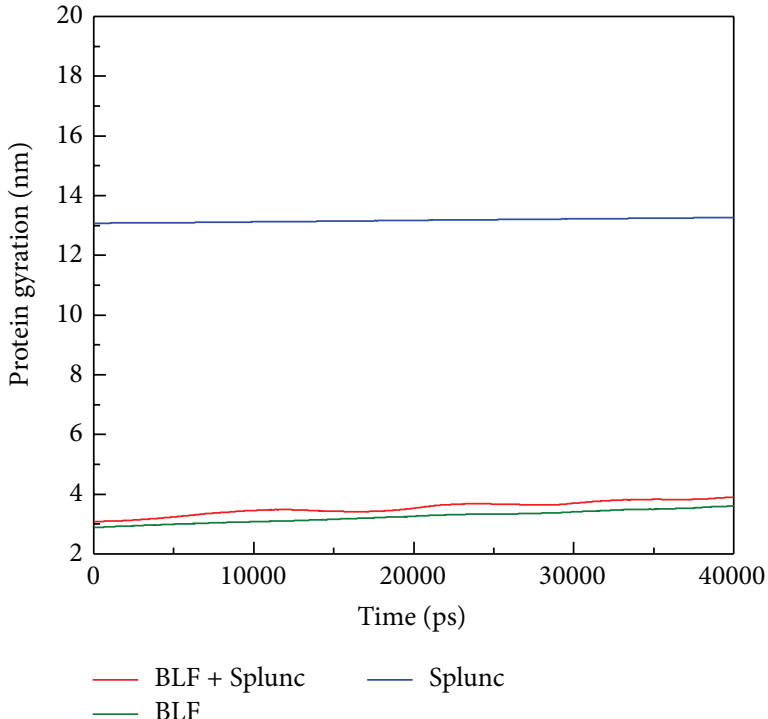

(a)

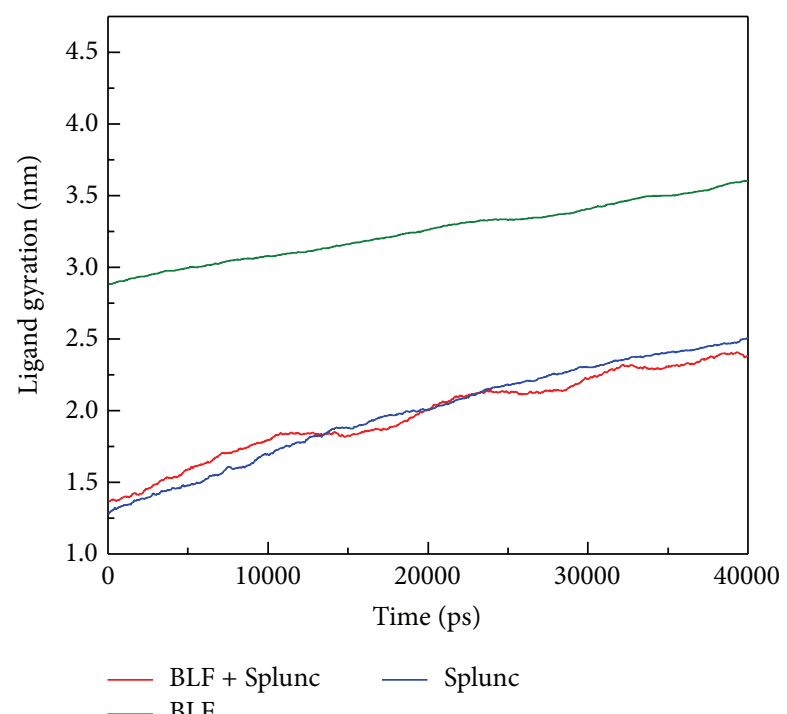

(b)

FIGURE 9: Radius of gyration analysis to measure the compactness. (a) Protein structure of BLF, SPLUNC, and protein-protein complex (BLF and SPLUNC). (b) Lipopolysaccharide.

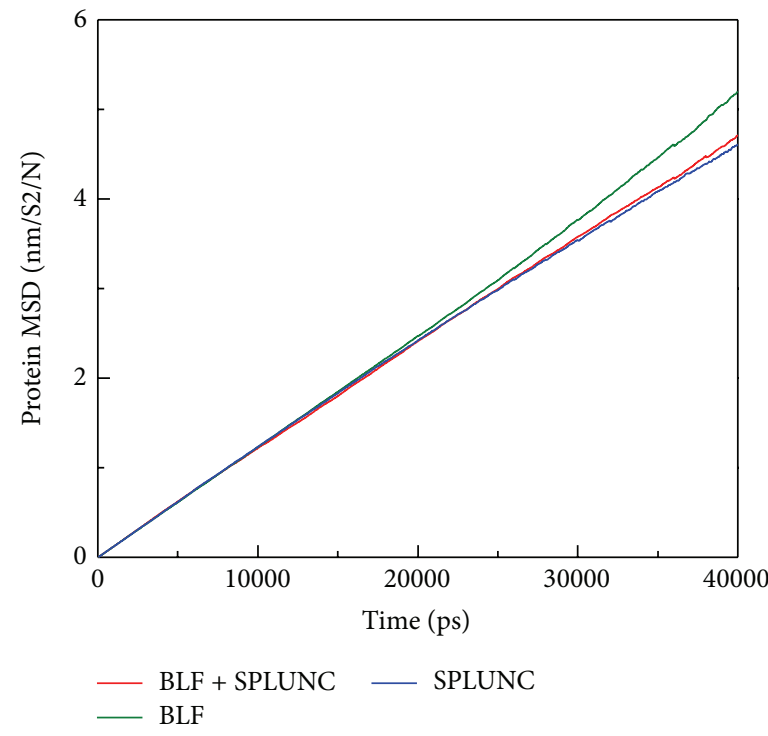

(a)

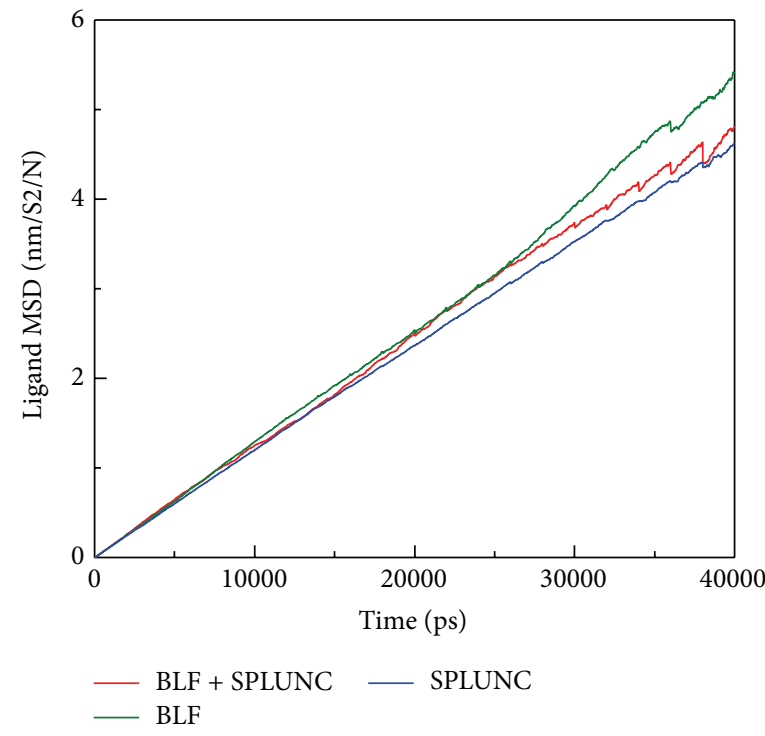

(b)

FIgURE 10: Mean square displacement (MSD) of atoms for atom migration assay. (a) Protein structure of BLF, SPLUNC, and protein-protein complex (BLF and SPLUNC). (b) Lipopolysaccharide.

University (ASIA100-CMU-2, ASIA101-CMU-2, and 102ASIA-07), and China Medical University Hospital (DMR103-058, DMR-103-001, and DMR-103-096). This study is also supported in part by Taiwan Department of Health Clinical Trial and Research Center of Excellence (DOH102TD-B-111-004) and Taiwan Department of Health Cancer Research Center of Excellence (MOHW103-TD-B-11103).

\section{References}

[1] L. Bingle and C. D. Bingle, "Distribution of human PLUNC/BPI fold-containing (BPIF) proteins," Biochemical Society Transactions, vol. 39, no. 4, pp. 1023-1027, 2011.

[2] Y. P. Di, "Functional roles of SPLUNC1 in the innate immune response against Gram-negative bacteria," Biochemical Society Transactions, vol. 39, no. 4, pp. 1051-1055, 2011. 
[3] Y. Liu, M. E. Di, H. W. Chu et al., "Increased susceptibility to pulmonary Pseudomonas infection in Spluncl knockout mice," The Journal of Immunology, vol. 191, no. 8, pp. 4259-4268, 2013.

[4] Y. A. Tsou, C. M. Chen, T. C. Lin et al., "Decreased SPLUNC1 expression is associated with Pseudomonas infection in surgically treated chronic rhinosinusitis patients who may require repeated sinus surgery," Laryngoscope, vol. 123, no. 4, pp. 845$851,2013$.

[5] F. Gally, Y. P. Di, S. K. Smith et al., "SPLUNC1 promotes lung innate defense against mycoplasma pneumoniae infection in mice," American Journal of Pathology, vol. 178, no. 5, pp. 21592167, 2011.

[6] Y. Liu, J. A. Bartlett, M. E. Di et al., "SPLUNC1/BPIFAl contributes to pulmonary host defense against Klebsiella pneumoniae respiratory infection," The American Journal of Pathology, vol. 182, no. 5, pp. 1519-1531, 2013.

[7] L. Gakhar, J. A. Bartlett, J. Penterman et al., "PLUNC is a novel airway surfactant protein with anti-biofilm activity," PLOS ONE, vol. 5, no. 2, Article ID e9098, 2010.

[8] A. Garcia-Caballero, J. E. Rasmussen, E. Gaillard et al., "SPLUNC1 regulates airway surface liquid volume by protecting $\mathrm{ENaC}$ from proteolytic cleavage," Proceedings of the National Academy of Sciences of the United States of America, vol. 106, no. 27, pp. 11412-11417, 2009.

[9] C. J. Britto, Q. Liu, D. R. Curran et al., "Short palate, lung, and nasal epithelial clone-1 is a tightly regulated airway sensor in innate and adaptive immunity," American Journal of Respiratory Cell and Molecular Biology, vol. 48, no. 6, pp. 717-724, 2013.

[10] G. McGillivary and L. O. Bakaletz, "The multifunctional host defense peptide SPLUNC1 is critical for homeostasis of the mammalian upper airway," PLoS ONE, vol. 5, no. 10, Article ID e13224, 2010.

[11] M. Sinha, S. Kaushik, P. Kaur, S. Sharma, and T. P. Singh, "Antimicrobial lactoferrin peptides: the hidden players in the protective function of a multifunctional protein," International Journal of Peptides, vol. 2013, Article ID 390230, 12 pages, 2013.

[12] L. H. Vorland, H. Ulvatne, J. Andersen et al., "Lactoferricin of bovine origin is more active than lactoferricins of human, murine and caprine origin," Scandinavian Journal of Infectious Diseases, vol. 30, no. 5, pp. 513-517, 1998.

[13] H.-L. Chen, C.-C. Yen, C.-Y. Lu, C.-H. Yu, and C.-M. Chen, "Synthetic porcine lactoferricin with a 20-residue peptide exhibits antimicrobial activity against Escherichia coli, Staphylococcus aureus, and Candida albicans," Journal of Agricultural and Food Chemistry, vol. 54, no. 9, pp. 3277-3282, 2006.

[14] J. S. Kim, M. B. Ellman, D. Yan et al., "Lactoferricin mediates anti-inflammatory and anti-catabolic effects via inhibition of IL-1 and LPS activity in the intervertebral disc," Journal of Cellular Physiology, vol. 228, no. 9, pp. 1884-1896, 2013.

[15] H. Ulvatne, H. H. Haukland, Ø. Olsvik, and L. H. Vorland, "Lactoferricin B causes depolarization of the cytoplasmic membrane of Escherichia coli ATCC 25922 and fusion of negatively charged liposomes," FEBS Letters, vol. 492, no. 1-2, pp. 62-65, 2001.

[16] M. B. Strøm, B. Erik Haug, Ø. Rekdal, M. L. Skar, W. Stensen, and J. S. Svendsen, "Important structural features of 15-residue lactoferricin derivatives and methods for improvement of antimicrobial activity," Biochemistry and Cell Biology, vol. 80, no. 1, pp. 65-74, 2002.

[17] A. J. Psaltis, P.-J. Wormald, K. R. Ha, and L. W. Tan, "Reduced levels of lactoferrin in biofilm-associated chronic rhinosinusitis," Laryngoscope, vol. 118, no. 5, pp. 895-901, 2008.
[18] S. Seshadri, D. C. Lin, M. Rosati et al., "Reduced expression of antimicrobial PLUNC proteins in nasal polyp tissues of patients with chronic rhinosinusitis," Allergy, vol. 67, no. 7, pp. 920-928, 2012.

[19] C. Y. Chen, "A novel integrated framework and improved methodology of computer-aided drug design," Current Topics in Medicinal Chemistry, vol. 13, no. 9, pp. 965-988, 2013.

[20] P.-C. Lin, P.-Y. Liu, S.-Z. Lin, and H.-J. Harn, "Angelica sinensis: a Chinese herb for brain cancer therapy," BioMedicine, vol. 2, no. 1, pp. 30-35, 2012.

[21] F.-J. Tsai, "Rare diseases: a mysterious puzzle," BioMedicine, vol. 3, no. 2, p. 65, 2013.

[22] Y.-M. Chang, B. K. Velmurugan, W.-W. Kuo et al., "Inhibitory effect of alpinate Oxyphyllae fructus extracts on Ang IIinduced cardiac pathological remodeling-related pathways in H9c2 cardiomyoblast cells," BioMedicine, vol. 3, no. 4, pp. 148152,2013

[23] S.-C. Hsu and J.-G. Chung, "Anticancer potential of emodin," BioMedicine, vol. 2, no. 3, pp. 108-116, 2012.

[24] T.-Y. Ho, H.-Y. Lo, C.-C. Li, J.-C. Chen, and C.-Y. Hsiang, "In vitro and in vivo bioluminescent imaging to evaluate antiEscherichia coli activity of Galla Chinensis," BioMedicine, vol. 3, no. 4, pp. 160-166, 2013.

[25] T.-T. Chang, M.-F. Sun, H.-Y. Chen et al., "Screening from the world's largest TCM database against H1N1 virus," Journal of Biomolecular Structure \& Dynamics, vol. 28, no. 5, pp. 773-786, 2011.

[26] C. Y.-C. Chen, "Virtual screening and drug design for PDE-5 receptor from traditional Chinese medicine database," Journal of Biomolecular Structure \& Dynamics, vol. 27, no. 5, pp. 627640, 2010.

[27] K. Y. Chen, S. S. Chang, and C. Y. Chen, "In silico identification of potent pancreatic triacylglycerol lipase inhibitors from traditional Chinese medicine," PLoS ONE, vol. 7, no. 9, Article ID e43932, 2012.

[28] K.-C. Chen, M.-F. Sun, S.-C. Yang et al., "Investigation into potent inflammation inhibitors from traditional Chinese medicine," Chemical Biology and Drug Design, vol. 78, no. 4, pp. 679-688, 2011.

[29] Y. A. Tsou, K. C. Chen, H. C. Lin, S. S. Chang, and C. Y. Chen, "Uroporphyrinogen decarboxylase as a potential target for specific components of traditional Chinese medicine: a virtual screening and molecular dynamics study," PLOS ONE, vol. 7, no. 11, Article ID e50087, 2012.

[30] C.-Y. Chen and C. Y.-C. Chen, "Insights into designing the dual-targeted HER2/HSP90 inhibitors," Journal of Molecular Graphics and Modelling, vol. 29, no. 1, pp. 21-31, 2010.

[31] S.-C. Yang, S.-S. Chang, and C. Y.-C. Chen, "Identifying HER2 inhibitors from natural products database," PLoS ONE, vol. 6, no. 12, Article ID e28793, 2011.

[32] S.-S. Chang, H.-J. Huang, and C. Y.-C. Chen, "Two birds with one stone? Possible dual-targeting H1N1 inhibitors from traditional Chinese medicine," PLoS Computational Biology, vol. 7, no. 12, Article ID e1002315, 2011.

[33] H. J. Huang, K. J. Lee, H. W. Yu et al., "Structure-based and ligand-based drug design for HER 2 receptor," Journal of Biomolecular Structure \& Dynamics, vol. 28, no. 1, pp. 23-37, 2010.

[34] C.-Y. Chen, Y.-H. Chang, D.-T. Bau et al., "Ligand-based dual target drug design for H1N1: swine flu-a preliminary first study," Journal of Biomolecular Structure \& Dynamics, vol. 27, no. 2, pp. 171-178, 2009. 
[35] C. Y. Chen, "Mechanism of BAG1 repair on Parkinson's diseaselinked DJ1 mutation," Journal of Biomolecular Structure \& Dynamics, vol. 30, no. 1, pp. 1-12, 2012.

[36] K.-C. Chen, K.-W. Chang, H.-Y. Chen, and C. Y.-C. Chen, "Traditional Chinese medicine, a solution for reducing dual stroke risk factors at once?" Molecular BioSystems, vol. 7, no. 9, pp. 2711-2719, 2011.

[37] K. C. Chen, S. S. Chang, H. J. Huang et al., "Three-in-one agonists for PPAR-alpha, PPAR-gamma, and PPAR-delta from traditional Chinese medicine," Journal of Biomolecular Structure \& Dynamics, vol. 30, no. 6, pp. 662-683, 2012.

[38] S.-S. Chang, H.-J. Huang, and C. Y.-C. Chen, "High performance screening, structural and molecular dynamics analysis to identify H1 inhibitors from TCM Database@Taiwan," Molecular BioSystems, vol. 7, no. 12, pp. 3366-3374, 2011.

[39] P.-C. Chang, J.-D. Wang, M.-M. Lee et al., "Lose weight with traditional Chinese medicine? Potential suppression of fat mass and obesity-associated protein," Journal of Biomolecular Structure \& Dynamics, vol. 29, no. 3, pp. 471-483, 2011.

[40] C. Y.-C. Chen, "Weighted equation and rules-a novel concept for evaluating protein-ligand interaction," Journal of Biomolecular Structure \& Dynamics, vol. 27, no. 3, pp. 271-282, 2009.

[41] T.-Y. Tsai, K.-W. Chang, and C. Y.-C. Chen, "IScreen: world's first cloud-computing web server for virtual screening and de novo drug design based on TCM database@Taiwan," Journal of Computer-Aided Molecular Design, vol. 25, no. 6, pp. 525-531, 2011.

[42] C. Y.-C. Chen, “TCM Database@Taiwan: the world’s largest traditional Chinese medicine database for drug screening In Silico," PLoS ONE, vol. 6, no. 1, Article ID e15939, 2011.

[43] I. C. Chou, W.-D. Lin, C.-H. Wang et al., "Möbius syndrome in a male with XX/XY mosaicism," BioMedicine, vol. 3, no. 2, pp. 102-104, 2013.

[44] C.-C. Lee, C.-H. Tsai, L. Wan et al., "Increased incidence of Parkinsonism among Chinese with $\beta$-glucosidase mutation in central Taiwan," BioMedicine, vol. 3, no. 2, pp. 92-94, 2013.

[45] D.-Y. Lin, F.-J. Tsai, C.-H. Tsai, and C.-Y. Huang, "Mechanisms governing the protective effect of $17 \beta$-estradiol and estrogen receptors against cardiomyocyte injury," BioMedicine, vol. 1, no. 1, pp. 21-28, 2011.

[46] W.-Y. Lin, H.-P. Liu, J.-S. Chang et al., "Genetic variations within the PSORS1 region affect Kawasaki disease development and coronary artery aneurysm formation," BioMedicine, vol. 3, no. 2, pp. 73-81, 2013.

[47] Y.-T. Chang, W.-D. Lin, Z.-N. Chin et al., "Nonketotic hyperglycinemia: a case report and brief review," BioMedicine, vol. 2, no. 2, pp. 80-82, 2012.

[48] Y. Zhang, "I-TASSER server for protein 3D structure prediction," BMC Bioinformatics, vol. 9, article 40, 2008.

[49] A. Roy, A. Kucukural, and Y. Zhang, "I-TASSER: a unified platform for automated protein structure and function prediction," Nature Protocols, vol. 5, no. 4, pp. 725-738, 2010.

[50] A. Roy, J. Yang, and Y. Zhang, "COFACTOR: an accurate comparative algorithm for structure-based protein function annotation," Nucleic Acids Research, vol. 40, pp. W471-W477, 2012.

[51] S. C. Lovell, I. W. Davis, W. B. Arendall III et al., "Structure validation by $\mathrm{C} \alpha$ geometry: $\phi, \psi$ and $\mathrm{C} \beta$ deviation," Proteins: Structure, Function and Genetics, vol. 50, no. 3, pp. 437-450, 2003.
[52] Accelerys, Discovery Studio 2.5, Accelrys Inc., San Diego, Calif, USA, 2009.

[53] S. A. Moore, B. F. Anderson, C. R. Groom, M. Haridas, and E. N. Baker, "Three-dimensional structure of diferric bovine lactoferrin at 2.8 Å resolution," Journal of Molecular Biology, vol. 274, no. 2, pp. 222-236, 1997.

[54] N. L. Allinger, "Conformational analysis. 130. MM2. A hydrocarbon force field utilizing V1 and V2 torsional terms," Journal of the American Chemical Society, vol. 99, no. 25, pp. 8127-8134, 1977.

[55] R. Chen and Z. Weng, "Docking unbound proteins using shape complementarity, desolvation, and electrostatics," Proteins: Structure, Function and Genetics, vol. 47, no. 3, pp. 281294, 2002.

[56] D. J. Diller and K. M. Merz Jr., "High throughput docking for library design and library prioritization," Proteins, vol. 43, no. 2, pp. 113-124, 2001.

[57] B. R. Brooks, R. E. Bruccoleri, B. D. Olafson et al., "CHARMM: a program for macromolecular energy, minimization, and dynamics calculations," Journal of Computational Chemistry, vol. 4, pp. 187-217, 1983.

[58] R. Fletcher and C. M. Reeves, "Function minimization by conjugate gradients," The Computer Journal, vol. 7, no. 2, pp. 149-154, 1964.

[59] W. H. Press, B. P. Flannery, S. A. Teukolsky, W. T. Vetterling, and H. Gould, "Numerical recipes, the art of scientific computing," American Journal of Physics, vol. 55, no. 1, pp. 90-91, 1987.

[60] J.-P. Ryckaert, G. Ciccotti, and H. J. C. Berendsen, "Numerical integration of the cartesian equations of motion of a system with constraints: molecular dynamics of n-alkanes," Journal of Computational Physics, vol. 23, no. 3, pp. 327-341, 1977.

[61] K. Maniar-Hew, C. C. Clay, E. M. Postlethwait et al., "Innate immune response to LPS in airway epithelium is dependent on chronological age and antecedent exposures," American Journal of Respiratory Cell and Molecular Biology, vol. 49, no. 5, pp. 710720, 2013.

[62] P. Manzoni, L. Decembrino, I. Stolfi et al., "Lactoferrin and prevention of late-onset sepsis in the pre-term neonates," Early Human Development, vol. 86, pp. 59-61, 2010.

[63] Y. A. Tsou, M. T. Peng, Y. F. Wu et al., "Decreased PLUNC expression in nasal polyps is associated with multibacterial colonization in chronic rhinosinusitis patients," European Archives of Oto-Rhino-Laryngology, vol. 271, no. 2, pp. 299-304, 2013.

[64] S. Sánchez-Gómez, B. Japelj, R. Jerala et al., "Structural features governing the activity of lactoferricin-derived peptides that act in synergy with antibiotics against Pseudomonas aeruginosa in vitro and in vivo," Antimicrobial Agents and Chemotherapy, vol. 55, no. 1, pp. 218-228, 2011. 


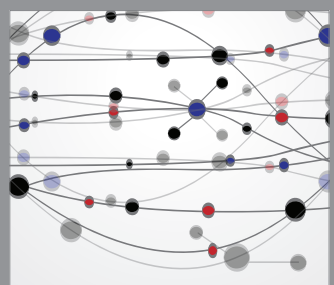

The Scientific World Journal
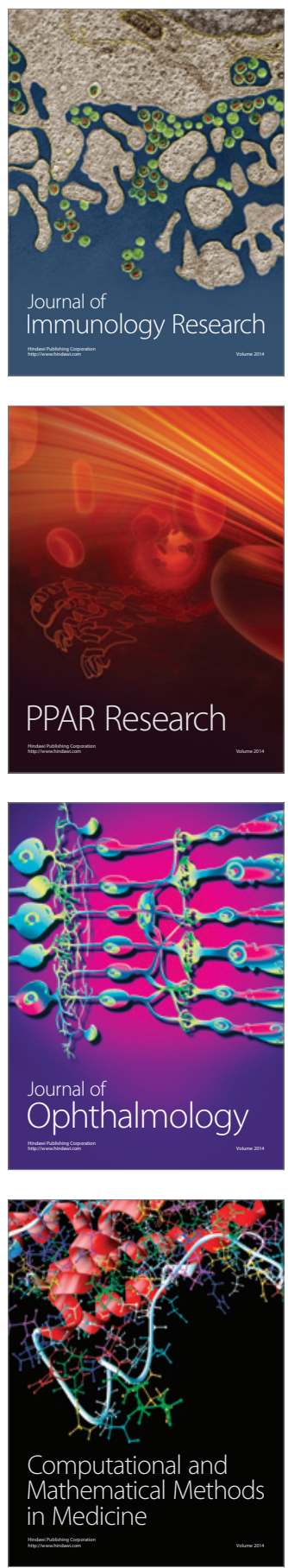

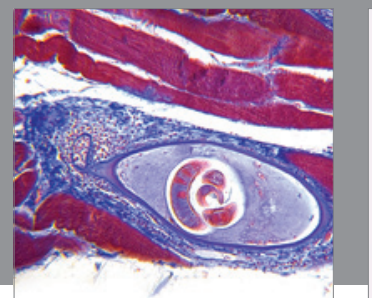

Gastroenterology

Research and Practice
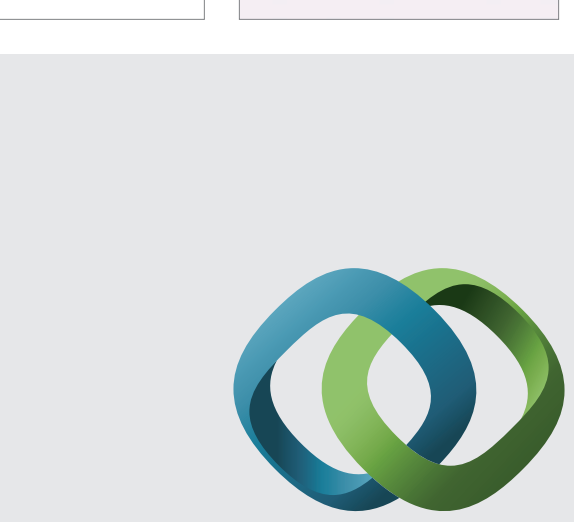

\section{Hindawi}

Submit your manuscripts at

http://www.hindawi.com
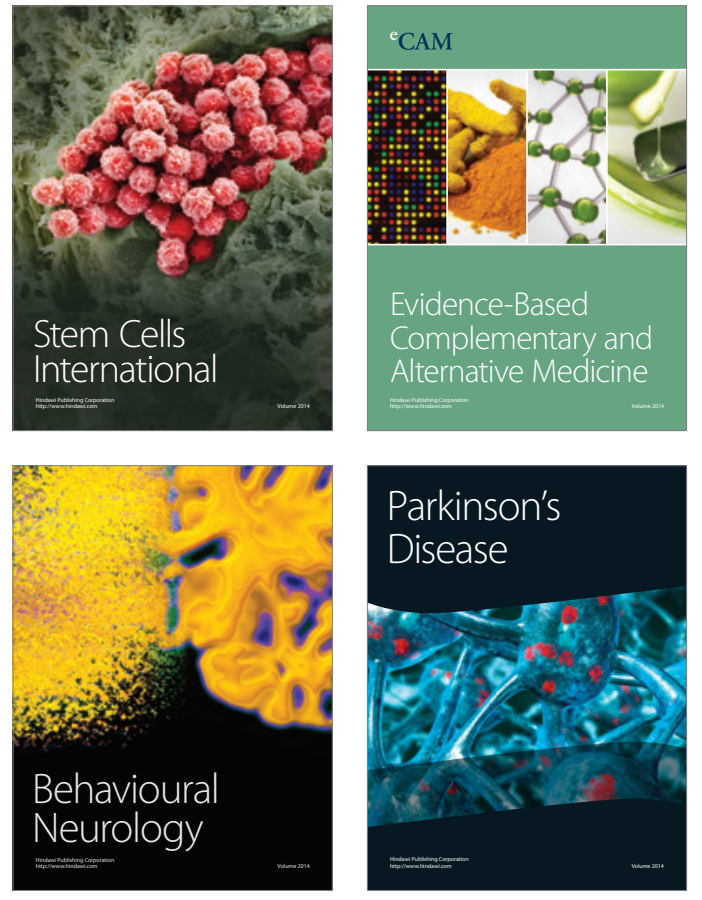
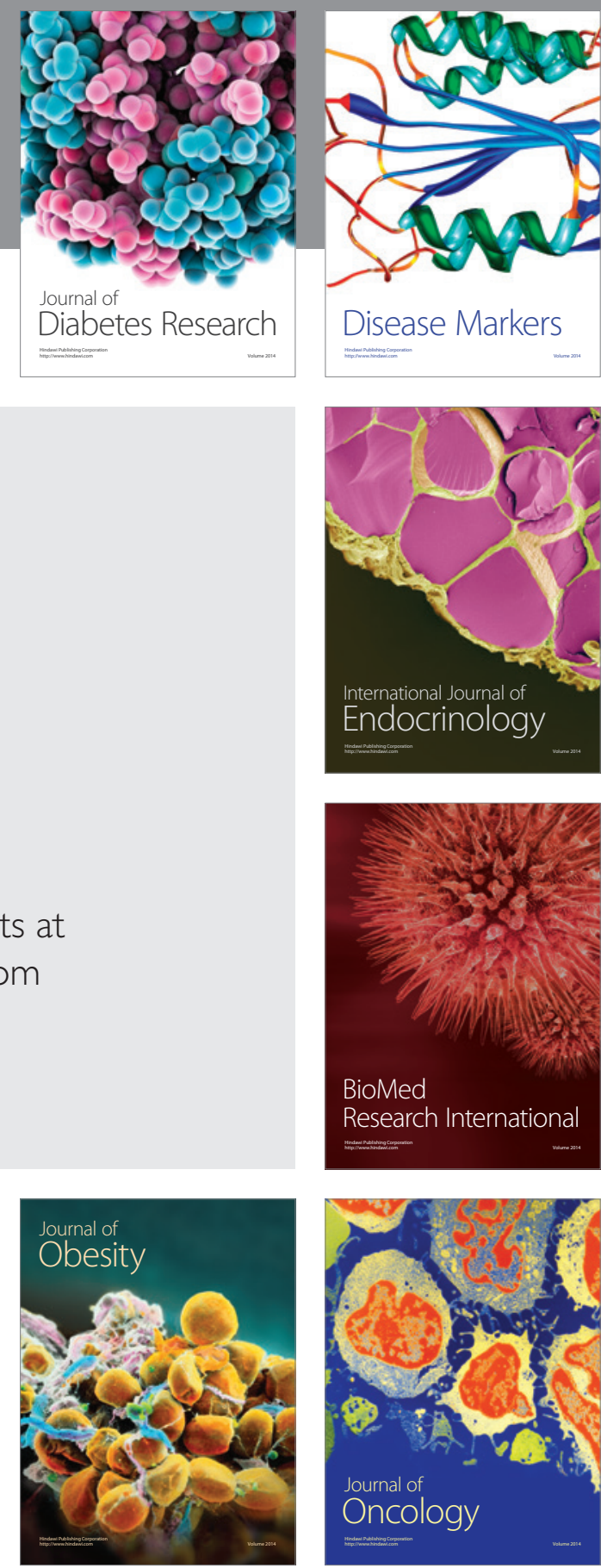

Disease Markers
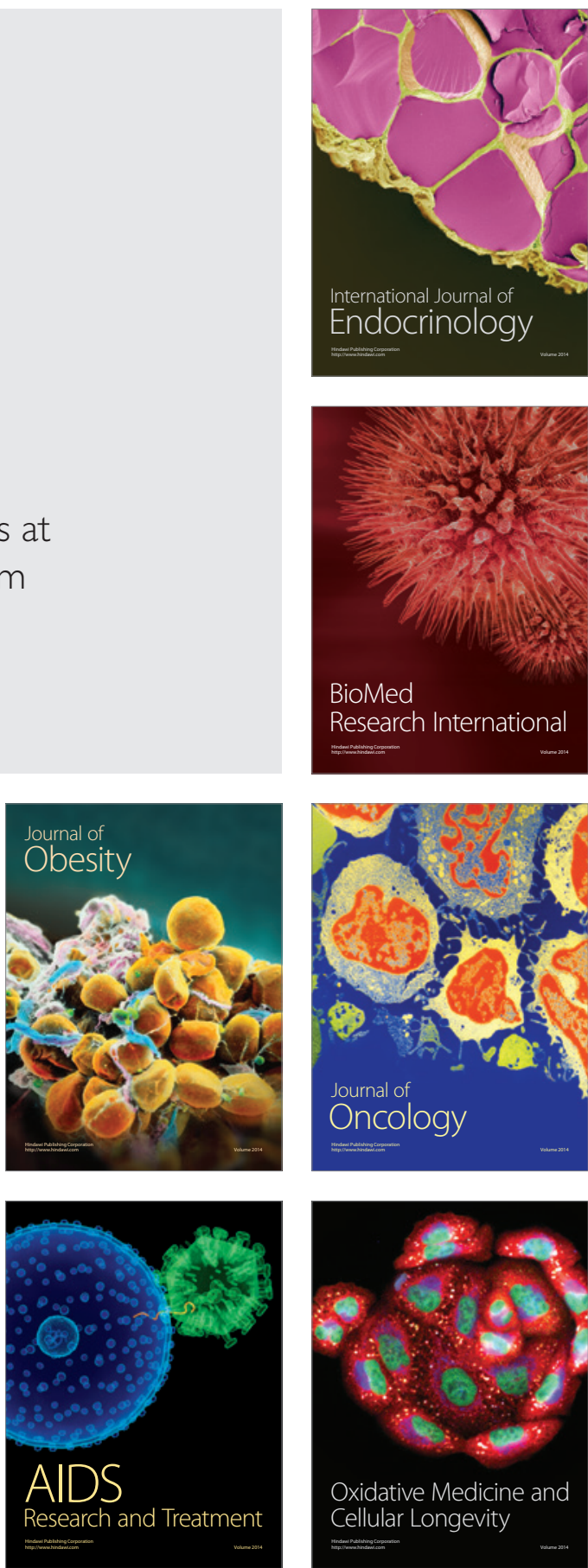